\title{
Das Konzept des Problemlösens als Modell zur Beschreibung und Erklärung literaturgeschichtlichen Wandels
}

\section{Uhrenvergleich}

Mit der Bezeichnung des Problem/Lösungs-Konzeptes als eines Beschreibungs- und Erklärungsmodells für literaturgeschichtlichen Wandel habe ich bereits eine Festlegung vorgenommen. >Problem terminus technicus geworden, dem eine bestimmte Verwendungsweise (>Bedeutung () innerhalb einer bestimmten Fachsprache zugeordnet ist. Diese Verwendungsweise ist zwar initial an die innerhalb der Alltagssprache angelehnt (aus ihr >entlehnt $<$ ), wird mit der Prägung eines Terminus aber von ihr abgekoppelt. ${ }^{1}$ Das hat unter anderem zur Folge, daß Bedeutungen, die dem Wort >Problem nicht gegen die heuristische Konzeption des Problem(lösen)s ins Feld geführt werden können (sondern allenfalls - mit einer guten Alternative im Gepäck - gegen die Wahl des Zentralbegriffs). Denn in dieser Konzeption ist >Problem ders als der alltagssprachliche Begriff nicht zwingend eine krisenhafte Erfahrung, ${ }^{2}$ sondern die eine Seite in einer erklärenden Relation von zwei Sachverhalten. Mit anderen Worten: In der Tat kann ich alles als Problem

1 Damit wende ich mich gegen Dirk Werles (»Modelle einer literaturwissenschaftlichen Problemgeschichte«, in: Jahrbuch der deutschen Schillergesellschaft 50 [2006], S. 478-498, hier S. 481 u. 496) ausdrückliche Befürwortung eines umgangssprachlichen Problembegriffs. (Dieser hätte u. a. die unpraktische Implikation, die in einem literarischen Werk reflektierten Probleme müßten dem Autor bewußt sein; vgl. z. B. ebd., S. 496: von der im Text vorhandenen Problemartikulation lasse sich auf die Autorintention schließen.) Auch fehlt mir daher das Verständnis für das feinsinnige Aushorchen der Bedeutungsnuancen zwischen >Problem/Lösung und >Frage/Antwort (vgl. Dirk Werle: »Frage und Antwort, Problem und Lösung«, in: Scientia Poetica 13 [2009], S. 255-303, hier S. 258-260). Es handelt sich vielmehr um ein (und dasselbe) Modell und verschiedene (wohl tatsächlich austauschbare) Begrifflichkeiten.

2 Vgl. Carlos Spoerhase: „Was ist kein Problem?«, in: Scientia Poetica 13 (2009), S. 318-328, hier S. 325 u. S. 327f. 
beschreiben bzw. »im Problemvokabular reformulieren « ${ }^{3}$ wenn ich nur will, das heißt: wenn ich mir davon einen wie auch immer gearteten Erklärungswert für etwas von mir als erklärungsbedürftig Erachtetes verspreche. Die Frage lautet also nicht: Wann ist ein Sachverhalt »treffend ${ }^{4}$ (d. i. nach unserer alltagssprachlichen Intuition stimmig) als Problem beschrieben? Sondern: Wann brauche ich den betreffenden Sachverhalt für eine erklärende Relation und muß ihn zu diesem Zweck als heuristische Einheit konzipieren (und nenne sie deshalb meinem Modell gemäß ein Problem)? Der Vorteil der universellen Applizierbarkeit (oder richtiger: zweckgebundenen Einstellbarkeit) des Konzepts >Problem $<$ kann nicht durch den Hinweis, daß dann »die Zahl der potentiell relevanten Probleme sich ins Unüberschaubare steiger[e],$^{5}$ einfach in einen Nachteil umgemünzt werden. Eine literaturgeschichtliche Problemrelation hat es in der Praxis ja nie mit der Menge aller potentiell rekonstruierbaren Probleme (im Sinne des ralles kann als Problem reformuliert werden`) zu tun, sondern lediglich mit den wenigen, im Hinblick auf ein bestimmtes Explanandum als relevant erachteten, so daß die theoretisch gegebene, sunüberschaubare Vielfalt in der Praxis selten dramatische Formen annimmt.

Auch 〉Lösung`, die andere Seite der Relation, bezeichnet als instrumenteller Terminus natürlich nicht notwendig, daß das Problem gelöst (im Sinne von saufgelöst, beseitigt $\varsigma$ ) würde, sondern daß der betreffende Sachverhalt - irgendwie - auf das veranschlagte Problem >reagiert`, mit ihm `zusammenhängt<, durch es hervorgebracht wurde, sich irgendwie auf es >bezieht` oder: eine bestimmte >Funktion` im veranschlagten Problemkontext erfüllt. Entsprechend finden sich häufig entkonkretisierte Alternativbegriffe wie `Problemlösungsaktivität‘, >Problemreferenz` oder >Problemreflexion<. Im Sinne einer analytischen Technik für die literaturwissenschaftliche Praxis lassen sich außerdem »Problemformulierung, -erklärung und -lösung « ${ }^{6}$ sinnvoll unterscheiden: Die Rekonstruktion der literarischen Problemformulierung dient dann dem Nachweis, daß das veranschlagte Problem tatsächlich zu den relevanten Kontexten eines Textes gehört; die Rekonstruktion der in einem Text vorliegenden Problemerklärung liefert eine vertiefte Analyse der fremden Regelmäßig-

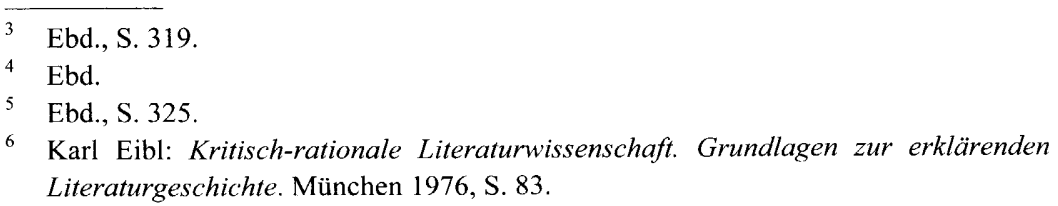

keitsannahmen (১Wissen ${ }^{7}$ ); und die Rekonstruktion der (spezifisch literarischen) Problemlösung buchstabiert die eigentliche Relation aus, indem sie zeigt, wie und warum die für ein bestimmtes Werk, einen Autor oder eine Epoche spezifische literarische Problembehandlung und -bearbeitung eine plausible ${ }^{8}$ Reaktion auf das veranschlagte Problem darstellt.

Vor allem in bezug auf die letzte dieser drei Kategorien möchte ich im folgenden einen etwas älteren Formulierungsvorschlag wieder in Erinnerung rufen, der mir zu Unrecht in Vergessenheit geraten zu sein scheint. Er kann die Weiterentwicklung der geistesgeschichtlichen >Problemgeschichte $<$ zu einer fachwissenschaftlichen Analytik markieren, hinter deren Errungenschaft man nicht mehr zurückfallen sollte.

\section{Literarisches >Verhalten}

An der Stelle der >Lösung steht in Paul Böckmanns Formgeschichte der deutschen Dichtung (1949) der Begriff der dichterischen Form. Böckmann übernimmt Wilhelm Diltheys Auffassung von Dichtung als einem »Organ des Weltverständnisses«, erhebt jedoch die genaue Beschreibung der historisch je spezifischen Beschaffenheit dieses `Organs` zur eigentlichen Aufgabe des Literaturwissenschaftlers (und grenzt sich damit von Diltheys psychologischem Interesse an den »seelischen Bedingungen und Voraussetzungen, die zum dichterischen Gestalten führen, « ab). ${ }^{9}$ Die Analyse von Dichtungen in ihrer Rolle als >Werkzeug ist für ihn gleichbedeutend mit einer Analyse der dichterischen `Form ^ oder `Gestalt々. Damit ist ein integrativer Formbegriff angesprochen, wie er etwa in der Tradition der >symbolischen Form` nach Ernst Cassirer (auf den Böckmann sich bezieht ${ }^{10}$ ) zum gängigen Ideenhaushalt der Zeit gehörte. In Böckmanns Analysen umschließt er sowohl die ‘äußere< Form (wie etwa Versmaß, Gattungskonventionen u. ä.) als auch deren konkrete sprachli-

Vgl. ebd., S. 50; vgl. auch Eibls Beitrag in diesem Band.

8 Plausibilität hier als Terminus für >Angepaßtheit< im Rahmen einer evolutionstheoretischen Konzeption von kulturellem Wandel; vgl. Niklas Luhmann: »Gesellschaftliche Struktur und semantische Tradition«, in: ders.: Gesellschaftsstruktur und Semantik. Studien zur Wissenssoziologie der modernen Gesellschaft. Frankfurt a. M. 1993, [zuerst 1980], Bd. 1, S. 9-71, hier S. 49.

9 Paul Böckmann: Formgeschichte der deutschen Dichtung, Bd. 1: Von der Sinnbildsprache zur Ausdruckssprache. Der Wandel der literarischen Formensprache vom Mittelalter zur Neuzeit. Hamburg 1949, S. 23.

10 Ebd., S. 27. 
che `Füllung ` in stilistischer und inhaltlicher Hinsicht. ${ }^{11}$ Böckmann bringt dieses holistische Konzept von >Form « mehrfach auf den Begriff eines je spezifischen >inneren Verhaltens`, das in den Werken zur Darstellung komme. ${ }^{12}$ Und die Rede von einem literarischen >Verhalten < ist bei Böckmann keine Metapher, sondern lediglich eine Generalisierung in einem grundsätzlich als konkret gedachten Modell: Die dichterische Formensprache sei »auf menschliche Grundverhaltungsweisen zurückzubeziehen $«{ }^{13}$ in ihr manifestierten sich »die Auffassungsformen des Menschlichen, in denen sich der Mensch über sich selbst verständigt, indem er die $\mathrm{ihm}$ bedeutsamen Züge des Daseins sinnfällig macht und sehen läßt. « ${ }^{14}$

Diese in Dichtung manifesten "Auffassungsformen des Menschlichen« werden von Böckmann radikal geschichtlich und - im Sinne von Werles Konzept der $>$ Zweigliedrigkeit ${ }^{15}-$ korrelativ gedacht. So steht das ganze Projekt einer Formgeschichte

unter der Hypothese, daß die Wandlungen des menschlichen Selbstverständnisses zugleich die Wandlungen der Formensprache nötig machen. Wenn die Dichtung nicht in begrifflicher Bestimmtheit, sondern in lebendiger Unmittelbarkeit vom Menschen spricht, dann muß ihre eigentliche Leistung darin bestehen, daß sie Auffassungsformen und Sehweisen entwickelt, mit denen sie die als bedeutsam empfundenen Züge des Daseins zur Darstellung bringen kann. Um das ihr aufgegebene Thema zu bewältigen, muß sie sich gestaltend bewähren. Und sie kann es nur in immer neuen individuellen Verwirklichungen, so daß von hier aus verständlich wird, warum sich alles Dichten in jeweils bestimmten und doch wechselnden Stilformen vollzieht. ${ }^{16}$

Hervorzuheben ist hier besonders der Gedanke der Bewährung, in welchem die Zweiheit von literarischem Text und außerliterarischem $>$ Kontext mitgedacht ist als Zweiheit von fokussiertem >System zu rekonstruierender, Selektionsdruck ausübender ,Umwelt $\ltimes$. Diesem evolutionstheoretischen Modell entsprechend erschöpfen sich die syn-

1 Werle: »Frage und Antwort« (wie Anm. 1), S. 255f., sortiert Fragen nach der dichterischen Form grundlos aus und ordnet die problemorientierte Analyse einer allgemeinen Ideengeschichte unter, $d . h$. schränkt sie auf die Befassung mit literarischen >Themen und Motiven < ein; vgl. auch die ähnlich gelagerte Kritik an Werle bei Matthias Löwe: »Implizität. Über ein praktisches Problem von Literaturgeschichte als Problemgeschichte (anhand von drei Beispielen)«, in: Scientia Poetica 13 (2009), S. 304-317, hier S. 307.

12 Vgl. Böckmann: Formgeschichte (wie Anm. 9), S. 61-65 u. pass.

13 Ebd., S. 65.

14 Ebd., S. 27

15 Werle: »Frage und Antwort« (wie Anm. 1), S. 256.

16 Böckmann: Formgeschichte (wie Anm. 9), S. $27 \mathrm{f}$ chronen Querschnitte durch die deutsche Literaturgeschichte, die Böckmann in seiner Formgeschichte vornimmt, nicht in einem Abklatsch üblicher Epocheneinteilung plus Bestimmung des jeweils zeitüblichen Stils, sondern sind darum bemüht, auch und gerade die Entstehung, den Wandel, Umbau oder das Unplausibelwerden einzelner Stile in möglichst kleinen Schritten nachzuvollziehen. Bei der Bestimmung des außerliterarischen Problemdrucks bleibt Böckmann vergleichsweise keusch, versäumt es aber nie, wenigstens durch einige Stichworte den geschichtlichen Rahmen abzustecken, innerhalb dessen er den betrachteten Wandel von Dichtung (und Poetik) motiviert sieht.

Böckmanns reaktives Modell aus literarischem >Verhalten ‘ und historischer >Situation « überwindet - zumindest konzeptionell - den impliziten Mimetismus früherer problemgeschichtlicher Ansätze. Auch Rudolf Unger ging schon von der Diltheyschen Charakterisierung der Dichtung als »Lebensdeutung $«{ }^{17}$ aus, führte diesen Gedanken jedoch weiter zur »Auffassung der Literatur als einer Spiegelung der Entwicklung sachlicher Probleme, und demgemäß der Literaturgeschichte als Problemgeschichte ${ }^{18}{ }^{18}$ Hier also werden System und Umwelt, Text und Kontext als prinzipiell kongruent gedacht; die Welt des Tatsächlichen, Sachlichen, Lebenswirklichen, die ins Konzept mit hinein zu nehmen zu Ungers großen Verdiensten zählt, erscheint hier nur als mimetische (und damit eigentlich redundante) Referenzstruktur. Erst Böckmann stößt zu einem wahrhaft zweigliedrigen Konzept vor, indem er zwischen Text und Kontext etwas abstrakter ein Passungsverhältnis postuliert, das nicht notwendig ein Abbildungsverhältnis sein muß. Die Anthropomorphisierung des Textes zum sich (gegenüber bestimmten kontextuellen Rahmenbedingungen) >verhaltenden` Organismus bleibt dabei stets rückbeziehbar auf die tatsächlich hinter dem Text stehenden handelnden Subjekte der literarischen Kommunikation und deren sowohl bewußt intendierte als auch unbewußte oder unkontrollierte kommunikative Handlungen.

Der Anschluß an Diltheys Organon-Konzeption und die Annahme, daß Dichtung generell historisch spezifische »Auffassungsformen des Menschlichen« darstelle, mag freilich gewisse Empfindlichkeiten in unse-

17 Rudolf Unger: »Literaturgeschichte als Problemgeschichte. Zur Frage geisteshistorischer Synthese, mit besonderer Beziehung auf Wilhelm Dilthey [1924]«, in: ders: Aufsätze zur Prinzipienlehre der Literaturgeschichte. Berlin 1929, S. 137-170, hier S. 144.

18 Ebd.; Hervorhebungen K. M. 
rem Fach berühren. Denn in einem sind wir uns ja alle einig: Überzeitliches ist pfui! - Aber warum eigentlich?

\section{Das Gespenst der Überzeitlichkeit}

So mag es z. B. recht schal wirken, wenn eine Interpretation von Gottfried Kellers Romeo und Julia auf dem Dorfe mit dem Fazit endet, die Novelle beziehe sich auf das ewige Problem der unbedingten Liebe in einer bedingten Welt. ${ }^{19}$ Nicht nur wird allen, die sich einmal mit der Historizität von Liebeskonzeptionen befaßt haben, die pauschale Rede von sunbedingter Liebeく etwas aufstoßen müssen. Auch daß die bis ins konkrete Detail satirisch ausgearbeiteten Verhältnisse in Seldwyla in dieser Interpretation lediglich als kontingente Manifestation einer >bedingten Welt $<$ in den Blick geraten, mag zumindest den Keller-Spezialisten etwas unbefriedigt lassen. Der zitierte Interpret verweist zu seiner Rechtfertigung auf die einleitenden Worte des Erzählers, die uns auf eine Geschichte vorbereiten, die

zu erzählen [...] eine müßige Erfindung sein [würde], wenn sie nicht auf einem wahren Vorfall beruhte, zum Beweise, wie tief im Menschenleben jede der schönen Fabeln wurzelt, auf welche ein großes Dichterwerk gegründet ist. Die Zahl solcher Fabeln ist mäßig, gleich der Zahl der Metalle, aber sie ereignen sich immer wieder auf's Neue mit veränderten Umständen und in der wunderlichsten Verkleidung. ${ }^{20}$

\section{Ganz ähnlich könnte man Heinrich Heines Gedicht}

Ein Jüngling liebt ein Mädchen,

Die hat einen andern erwählt;

Der andre liebt eine andre,

Und hat sich mit dieser vermählt.

Das Mädchen heiratet aus Ärger

Den ersten besten Mann,

Der ihr in den Weg gelaufen;

Der Jüngling ist übel dran.

Es ist eine alte Geschichte,

Doch bleibt sie immer neu;

19 Michael Schmitz: „Um Liebe, Leben und Tod. Zur Struktur und Problemreferenz von Gottfried Kellers Romeo und Julia auf dem Dorfe«, in: Wirkendes Wort 52 (2002), S. 67-80.

20 Gottfried Keller: Sämtliche Werke in sieben Bänden, hg. v. Thomas Böning, Gerhard Kaiser und Dominik Müller. Frankfurt a. M. 1985-96, Bd. 4, S. 69.
Und wem sie just passieret,

Dem bricht das Herz entzwei. ${ }^{21}$

anführen und behaupten, Heine habe damit weiter nichts als das Problem der nichtgarantierten Gegenseitigkeit der Liebesempfindung bearbeitet in der Tat ein leidiges Problem in allen Zeiten und Kulturen. Und ich glaube durchaus, daß es zahllose Gedichte in allen Sprachen der Welt gibt, über die sich genau dies sagen ließe: daß sie einfach nur das allgemeine \Liebesglück und Liebesleid` besingen; Liebesgedichte, die weiter nichts sind als eben Liebesgedichte. Allerdings sind das in der Regel nicht diejenigen Gedichte, mit denen sich der Literaturwissenschaftler bevorzugt befaßt. Der Grund, weshalb Interpretationen wie die hier angedachten irgendwie inadäquat wirken, liegt nicht darin, daß die damit angenommene Sorte Literatur inexistent oder gar theoretisch ausgeschlossen wäre, sondern darin, daß sie Antworten auf eine nicht gestellte Frage liefern. Den Literaturhistoriker interessiert in der Regel nicht so sehr, warum das Thema Liebe in Literatur überhaupt und zu allen Zeiten auftaucht (das scheint ihm selbstverständlich), sondern eher, welche spezifischen Formen der Thematisierung zu bestimmten Zeiten in die sgepflegte Semantik eingehen; welche spezifische Funktion das Liebesmotiv innerhalb eines bestimmten Werkzusammenhangs (wie z. B. Kellers Novellenoder Heines Liedersammlung) erfüllt; oder warum eine bestimmte Form der Thematisierung plötzlich neu erfunden oder besonders populär wird, kurz: er ist in der Regel am Wandel stärker als an der Konstanz interessiert.

Das ist nicht zwingend. Von einem literaturtheoretischen Standpunkt aus wäre auch eine ganz andere Fragestellung denkbar, etwa: Warum werden (zu allen Zeiten und in allen Kulturen) Lieder und Gedichte über die Liebe gemacht und rezipiert, statt es bei einer sang- und klanglosen Erfüllung der Reproduktionsfunktion zu belassen? Warum z. B. schreiben Pennäler Liebesgedichte, schwelgen (nicht nur) Verliebte in der Liebeslyrik von Volks- und Popmusik, besingt eine Eipo (Neuguinea) eine erotische Erfahrung ${ }^{22}$ und ein(e) Gond (Zentralindien) die Sehnsucht nach

${ }_{21}$ Heinrich Heine: Sämtliche Schriften, hg. v. Klaus Briegleb. 6 Bde. München $1968-$ 76, Bd. 1, S. 90 f.

22 "Bruder des wirye-Vogels, Bruder des cang-Baums, Bruder des dikle-Baums. / Der wirye-Vogel liegt mir nah am Nabel. / Meine Falle ist dabei zu quetschen, meine Falle ist dabei zu fangen. / Der wirye-Vogel bleibt hängen an meinem Nabel, / das cang-Holz liegt auf meiner Scham. / Nachdem ich den toktokana-Frosch [sc. das Grasröckchen] weggenommen habe, / nachdem ich den mokmokana-Frosch [dass.] weggenommen habe. / Auf der Scham liegend, am Nabel festhakend, hakt er sich 
der/dem Geliebten? ${ }^{23}$ Anders gesagt, warum ist Liebe vielleicht schon auf der biologischen Ebene ein >Problem $<$, dem ein bestimmtes (für den Literaturwissenschaftler nicht ganz uninteressantes) Verhalten korreliert? Ich will diese Frage hier nicht weiter verfolgen, sondern lediglich deutlich machen, daß das Abstellen auf überzeitliche Probleme nichts an und für sich Unsinniges, sondern lediglich durch die Art, wie heutige literaturhistorische Forschung in der Regel ihr Explanandum bildet, mehr oder weniger ausgeschlossen ist. Wenn Unger meint, es seien »vor allem [...] die elementaren Probleme des Menschenlebens, die großen, ewigen Rätsel- und Schicksalsfragen des Daseins, deren gestaltende Deutung den Kerngehalt alles Dichtens bildet «, ${ }^{24}$ dann mag man über seine Begriffe von >Kerngehalt` und >alles Dichtens` gewiß noch etwas länger nachdenken wollen, aber man begäbe sich mit diesem Nachdenken keineswegs in den Bereich des Absurden.

Ernster zu nehmende Schwierigkeiten, überzeitliche Bedingungen als beteiligte Faktoren mit in Betracht zu ziehen, scheinen mir eher daraus $\mathrm{zu}$ entstehen, daß der Status der historischen Erklärung dadurch unsicher wird bzw. einer genaueren Spezifizierung bedarf. Dazu ein Beispiel.

\section{Just-so stories versus Funktionsanalyse}

(Das Problem der Beliebigkeit)

Ein kluger Rezensent meines Buches Emotionalisierung ${ }^{25}$ zeigte Skepsis gegenüber meinem Vorschlag, die scherzhafte Sprechhaltung in der Literatur des deutschen Rokoko als Antwort auf das Problem des exponentialen Wissenszuwachses im Gelehrtenwesen des frühen 18. Jahrhunderts

mir fest.« Übers. nach Irenäus Eibl-Eibesfeld: Biologie des menschlichen Verhaltens Grundriß der Humanethologie. 3., überarb. und erw. Aufl. München-Zürich 1995, S. 951f. (nach einem Bericht von Volker Heeschen). Vgl. auch Karl Eibl: Die Entstehung der Poesie. Frankfurt a. M.-Leipzig 1995, S. 25, Fn. 44.

${ }_{23}$ $\mathrm{O}$ come, my body is alone, come laugh with me, come talk with me. / Bring mind to mind: clasp heart to heart. / What of the future? I care not for the past. / O come, beloved; come, laugh with me, / Come, talk with me. My body is alone.« Übers. nach: A World Treasury of Oral Poetry, ed. with an introduction by Ruth Finnegan. London-Bloomington 1978, S. 20.

24 Unger: »Literaturgeschichte als Problemgeschichte « (wie Anm. 17), S. 155.

25 Katja Mellmann: Emotionalisierung - Von der Nebenstundenpoesie zum Buch als Freund. Eine emotionspsychologische Analyse der Literatur der Aufklärungsepoche. Paderborn 2006 (Poetogenesis 4). und des damit verbundenen Professionalisierungsschubes aufzufassen. Er fragt ganz grundsätzlich,

weshalb es überhaupt eines historischen Bezugsproblems bedarf, um die literarischen Innovationen des 18. Jahrhunderts zu erklären, wenn man doch zuvor plausibel gemacht hat, dass sie auf anthropologischen Dispositionen beruhen. Das wäre so, als wollte man, nachdem man erklärt hat, weshalb wir Fettes und Süßes mögen, nach einem historischen Bezugsproblem suchen, um zu erklären, weshalb unsere Lebensmittelindustrie Fettes und Süßes massenhaft herstellt. Sie kann es einfach.

Ähnliches lässt sich von den Innovationen behaupten, die Mellmanns detaillierte Lektüren als historisch signifikant herausstellen: Nicht die Bedürfnisse haben sich geändert, sondern die kommunikationstechnischen Möglichkeiten ihrer Befriedigung. Eine schriftliche Scherzkultur etabliert sich, weil das Scherzen zu unserem anthropologischen Erbe gehört und in der Schriftkultur des 18. Jahrhunderts einen neuen kulturellen Lebensraum findet. ${ }^{26}$

Mit diesen Einwendungen richtet sich der Rezensent gegen die Wahl meines Explanandums. Die von mir untersuchten literarischen Wandlungsprozesse seien »nicht begründungspflichtig «, ${ }^{27}$ sondern erwartbare Manifestationen überzeitlicher Bedürfnisse im Zuge einer zunehmend »medienvermittelten Erlebniskultur ${ }^{28}$ - und meine historischen Erklärungen des Nichterklärungsbedürftigen folglich Scheinkonstruktionen.

Solche Scheinkonstruktionen fallen in den Bereich dessen, was man in der biologischen Evolutionstheorie gerne als just-so stories (nach Rudyard Kipling) bezeichnet: ätiologische Ad-hoc-Erklärungen, die entweder falsch oder unüberprüfbar sind, z. B. weil sie schon vom falschen Explanandum ausgehen. Die Adaptivität wird sozusagen generell vorausgesetzt, so daß, egal welches Element jeweils herausgegriffen wird, immer auch die Bestimmbarkeit eines Bezugsproblems erwartet (und dann nach gusto erfüllt) wird. Dasselbe gilt mutatis mutandis natürlich auch für alle Konzepte kultureller Evolution; und Warnungen, daß es zunächst einmal die Unwahrscheinlichkeit des vermuteten Explanandums herauszustellen gilt und daß es der Überprüfung bedarf, ob das Bezugsproblem richtig identifiziert (oder nur ad hoc eines erfunden) wurde, sind deshalb hier wie dort durchaus am Platze. Die biologisch verankerten Bedürfnisstrukturen im Menschen sind - wie die vom zitierten Rezensenten zu Recht beson-

\footnotetext{
26 Robert Vellusig: »Texte sind auch nur Menschen. Katja Mellmann über emotionale Attrappen und deren kulturelle Evolution«, in: IASLonline [15.05.2008], http:// www.iaslonline.de/index.php?vorgang id=2570, par. $38 \mathrm{f}$.

27 Ebd., par. 39.

28 Ebd., par. 41.
} 
ders hervorgehobenen medialen Entwicklungen - immer schon als ermöglichende Faktoren mit im Spiel, wenn der Literaturhistoriker mit der Arbeit beginnt, und daher bei der Eingrenzung des literaturgeschichtlichen Explanandums notwendig mit zu berücksichtigen.

Ich halte es indes noch immer für plausibel, daß der Zuwachs an verfügbarem Wissen eine Kultur der scherzhaften Distanznahme und spielerischen Wissensthesaurierung beförder ${ }^{29}$ und die fortschreitende Partikularisierung der individuellen Wissensbereiche eine ostentative Geselligkeitskultur unter Gelehrten hervorbringt, innerhalb derer die urplötzlich massenhaft ansteigende (und dann ebenso plötzlich wieder versiegende) Produktion scherzhafter Dichtung einen wichtigen, eigens institutionalisierten ${ }^{30}$ Teil ausmacht. Und mir scheint, daß man mit einer solchen These der Möglichkeit angemessen Rechnung trägt, daß gewisse Motive und Sprechweisen in der Rokokolyrik eine andere Funktion und Bedeutung haben als in ihren antiken Vorbildern oder anderen Vorläufern (wie etwa der mittelalterlichen und spätmittelalterlichen Lyrik oder der Bukolik des 17. Jahrhunderts). Meine Wahl des Explanandums ist also wesentlich motiviert durch die Intuition eines Funktionswandels zum Teil alter, aber auch erkennbar neu arrangierter und teilweise veränderter Dichtungsformen.

$\mathrm{Ob}$ ich mit dem von mir vorgeschlagenen Referenzproblem das richtige getroffen habe, sei dahingestellt. ${ }^{31}$ Wichtiger ist mir an dieser Stelle das Prinzip des Funktionswandels oder auch: der »Umdeutung«, wie es Marianne Willems (in einer unbedingt zu den >besten Beispielen historischen Bezugsproblemen fragenden Literaturwissenschaft zählen-

29 Vgl. auch Böckmann: Formgeschichte (wie Anm. 9), S. 472: Die Übernahme des >witzigen< Stilideals aus Frankreich sei motiviert durch »das neue Erkenntnisideal« der Aufklärung, durch das wauch die Dichtung sich vor eine neue Verantwortung gebracht sah und dem Anspruch der Erfahrungsnähe und Gedankenklarheit auf ihre Weise genug zu tun suchte.« Böckmann beschreibt mit diesem >Formprinzip des Witzes eine wichtige Vorstufe dessen, was ich unter dem Titel der >scherzhaften Sprechhaltung، besonders herauszustellen versucht habe.

30 Vgl. den sehr erhellenden Aufsatz von Wilhelm Voßkamp: "Gattungen als literarisch-soziale Institutionen«, in: Textsortenlehre - Gattungsgeschichte, hg. v. Walter Hinck. Heidelberg 1977, S. 27-44.

31 Einer meiner Anhaltspunkte ist die enge Verzahnung des dichterischen mit dem medikologischen Diskurs, der m. E. Aufschluß gibt über die (oder zumindest eine wichtige) Funktion der Scherzgedichte für die Zeitgenossen; vgl. Mellmann: Emotionalisierung (wie Anm. 25), S. 344-350. den Studie) sinnfällig beschrieben hat. ${ }^{32}$ Die durch die biologische Basis vorgegebenen Möglichkeiten werden nicht nur (gemäß den jeweiligen mediengeschichtlichen Bedingungen) quasi >im Leerlauf $\mathrm{s}^{33}$ aktualisiert das auch -, sondern müssen auf den jeweiligen soziokulturellen Kommunikationszusammenhang abgestimmt werden, das heißt: sie werden spezifisch semantisiert. Und dabei wird in der Regel an vorgängige >semantische Vorräte` umdeutend angeschlossen. Diese kulturellen Anpassungsoder Semantisierungsprozesse können je nach Fall unterschiedlich gravierender Art - und somit für den Literaturhistoriker unterschiedlich interessant - sein. Z. B. korreliert der Umstand, daß das Lied der Eipo-Frau speziell eine erotische Erfahrung (bzw. sogar nur einen bestimmten Ausschnitt derselben) thematisiert, vielleicht mit einer bestimmten Problemkonstellation (und damit zusammenhängenden erotischen Semantik) ihrer Kultur; zumindest für den völkerkundlichen Laien aber mag es ausreichend erscheinen, das Gedicht einfach als Liebesgedicht (ohne allzu viel soziokulturell bedingten semantischen $>$ Überschuß<) zu verstehen. ${ }^{34}$ Aber spätestens, wenn z. B. die biologische Basis der $>$ Limerenz $>{ }^{35}$ zur Codierung und Stabilisierung von Individualität eingesetzt wird, wie dies in den mitteleuropäischen Literaturen seit dem 18. Jahrhundert verstärkt (d. h. sinstitutionalisiert/) der Fall ist, wird es sich für den Literaturwissenschaftler lohnen, in dieser >Umdeutung`von Liebe ein literaturge-

32 Marianne Willems: Das Problem der Individualität als Herausforderung an die Semantik im Sturm und Drang. Studien zu Goethes Brief des Pastors zu $* * *$ an den neuen Pastor zu ***, Götz von Berlichingen und Clavigo. Tübingen 1995 (Studien und Texte zur Sozialgeschichte der Literatur 52).

33 Vgl. das evolutionspsychologische Konzept des "Organisationsmodus«, in welchem unsere biologische Grundausrüstung in Betrieb gehalten wird, nach John Tooby u. Leda Cosmides: „Does Beauty Build Adapted Minds? Towards an Evolutionary Theory of Aesthetics, Fiction, and the Arts «, in: SubStance. A Review of Theory and Literary Criticism 30 (2001), S. 6-27, hier S. 16. Dt. Übers.: »Schönheit und mentale Fitness. Auf dem Weg zu einer evolutionären Ästhetik«, in: Heuristiken der Literaturwissenschaft. Disziplinexterne Perspektiven auf Literatur, hg. v. Uta Klein, Katja Mellmann u. Steffanie Metzger. Paderborn 2006 (Poetogenesis 3), S. 217-243, hier S. 230.

34 Zumal es im Bericht heißt: »Liebeslieder dieser Art singt man auch, wenn man völlig allein ist, aus einer vergnügt-besinnlichen Stimmung heraus. Das Lied erscheint damit von sozialen Funktionen befreit.« Eibl-Eibesfeld: Biologie (wie Anm. 22), S. 952.

35 Begriffsprägung durch Dorothy Tennov: Love and Limerence. The Experience of Being in Love. New York 1979; eine gute evolutionspsychologische Bestimmung des psychischen Mechanismus als >attraction system Love. The Nature and Chemistry of Romantic Love. New York 2004. 
schichtlich relevantes Explanandum zu erblicken und die vermutete spezifische Problemkorrelation (Funktion) anhand der literarischen Problemformulierungen zu überprüfen und mit Hilfe außerliterarischer Kontexte genauer auszubuchstabieren. ${ }^{36}$

Die resultierende Erklärung dürfte freilich selten eine shinreichendeく Erklärung sein, denn komplexe Phänomene verdanken sich in aller Regel nicht einer einzigen Ursache, sondern einem ganzen Bündel kausalrelevanter Faktoren. Suspendiert man die verführerische Erwartung von Monokausalität und Nicht/Sondern-Erklärungen jedoch, können Problem/Lösungs-Relationen nach dem beschriebenen Modell - je nach Art des eingegrenzten Problems und der gewählten Kontextwissenschaft (wie beispielsweise Biologie, Gesellschaftsgeschichte, Medienwissenschaft) durchaus Erhellendes zur Erklärung eines komplexen kulturellen Phänomens (und damit zu einer erklärend vorgehenden Literaturwissenschaft) beitragen.

36 Solch ein Verfahren ist verschiedentlich als zirkulär bezeichnet worden (und wird es wohl noch häufiger werden). Ich erspare mir und meinen Lesern einen Abschnitt "V. Das Gespenst der Zirkularität«, indem ich auf Voßkamp: »Gattungen« (wie Anm. 30), S. 44, verweise: »Die Analyse der einzelnen Gattung setzt zugleich ihre genaue Bestimmung im jeweiligen literarischen Gesamtsystem einer Epoche und geschichtlichen Situation voraus. Dies ist methodologisch nur möglich, wenn man sich dem hermeneutischen Zirkel stellt [...].« 